\title{
Aeroacoustic Improvements to Fluidic Chevron Nozzles
}

\author{
Brenda Henderson ${ }^{*}$ and Kevin Kinzie ${ }^{\dagger}$ \\ NASA Langley Research Center, Hampton,VA, 23681 \\ Julia Whitmire ${ }^{\ddagger}$ and Amal Abeysinghe ${ }^{\S}$ \\ Goodrich Aerostructures, Chula Vista 91910
}

\begin{abstract}
Fluidic chevrons use injected air near the trailing edge of a nozzle to emulate mixing and jet noise reduction characteristics of mechanical chevrons. While previous investigations of "first generation" fluidic chevron nozzles showed only marginal improvements in effective perceived noise levels when compared to nozzles without injection, significant improvements in noise reduction characteristics were achieved through redesigned "second generation" nozzles on a bypass ratio 5 model system. The second-generation core nozzles had improved injection passage contours, external nozzle contour lines, and nozzle trailing edges. The new fluidic chevrons resulted in reduced overall sound pressure levels over that of the baseline nozzle for all observation angles. Injection ports with steep injection angles produced lower overall sound pressure levels than those produced by shallow injection angles. The reductions in overall sound pressure levels were the result of noise reductions at low frequencies. In contrast to the first-generation nozzles, only marginal increases in high frequency noise over that of the baseline nozzle were observed for the second-generation nozzles. The effective perceived noise levels of the new fluidic chevrons are shown to approach those of the core mechanical chevrons.
\end{abstract}

\section{Introduction}

$\mathrm{I}^{\mathrm{N}}$ $\mathrm{N}$ recent years, chevron mixing devices for jet noise reduction have advanced to the point that they are now in commercial service. Chevrons, or serrations, placed around the trailing edge of either the core or fan exhaust nozzle promote mixing of the jet flow and result in lower bulk jet velocity and lower noise. Mixing occurs by penetrating the chevron tip a small distance into the flow and producing streamwise vorticity from the pressure differential across the chevron. Mechanical chevrons are relatively simple to manufacture and install. However, as passive devices with a fixed geometry, they have the disadvantage of only being optimized for one flight condition. They cannot adapt to changes in the flow environment and adjustments cannot be made to compensate for changing flight operations or installation effects. In addition, thrust losses that occur while generating the mixing remain during cruise even though noise reduction is generally only needed during takeoff.

Active mixing techniques are particularly attractive as they have the potential to optimize jet noise reduction throughout flight operations and can be deployed only when noise reduction is necessary and accompanying performance penalties can be tolerated. One such active mixing technique is to inject air at the trailing edge of the exhaust nozzle in such a way as to generate similar vorticity and mixing characteristics as the mechanical chevron. These "fluidic chevrons" can be operated with different injection flow rates or injection location in a way that optimizes their performance for the given flight condition.

The key to successful noise reduction with chevron nozzles (mechanical or fluidic) is to reduce low frequency noise and not significantly increase high frequency noise ${ }^{1,2,3}$. For mechanical chevrons, the number of chevrons, the serration geometry, and the penetration depth as well as many other factors affect the acoustic radiation resulting from the chevron nozzle. Comparisons between numerical results and acoustic measurements indicate that some of

\footnotetext{
* Reasearcher, Aeroacoustics Branch, MS 166, Hampton, VA 23681.

${ }^{\dagger}$ Now at GE Energy, Acoustics Technical Leader, PGT Aerodynamic and Acoustics Technology, P.O. Box 648, GTTL 1236, Greenville, SC 29602.

‡ Staff Engineer, Acoustics Group, MZ 107N, Chula Vista, CA 91910.

${ }^{\S}$ Senior Engineer, Acoustics Group, MZ 107N, Chula Vista, CA 91910.
} 
the most aggressive mixers produce unacceptable levels of high frequency noise that completely offset any low frequency noise reduction when the effects of human perception of noise are taken into account.

The same potential problem occurs for fluidic chevrons. Henderson et $\mathrm{al}^{4}$. and Kinzie et $\mathrm{al}^{5}$. describe fluidic chevrons tested in the NASA Langley Jet Noise Laboratory. These fluidic chevrons were the first of their kind designed with small slots cut near the trailing edge nozzle to allow air to inject into the flow and promote mixing between the core and fan streams. Various injection parameters were considered including injection port count, injection pressure ratio, and injector orientation. These "first generation" experiments demonstrated that while low frequency noise reduction is possible, there is also the potential for significant levels of increased high frequency noise. While the reason for the high frequency noise could not be demonstrated conclusively, the augmented levels were generally attributed to details of the injector port design such as flow path and increased thickness of the core nozzle trailing edge. Henderson et $\mathrm{al}^{4}$. showed that the increase in noise could not be accounted for simply by the injector noise alone.

This paper reports on a "second generation" fluidic chevron nozzle design that incorporated lessons learned from the experiments of references 4 and 5 . For the second-generation design, more care was taken to design the internal flow path of the injectors so that the effects of injection angle could be determined. In addition, a special fabrication process was developed so that the nozzle trailing edge thickness was minimized everywhere.

\section{Experimental Approach}

The experiments were conducted in the Low Speed Aeroacoustics Wind Tunnel (LSAWT) at NASA Langley Research Center shown schematically in Fig. 1. The LSAWT is an in-draft wind tunnel that provides a free jet for forward flight effects that can reach Mach numbers up to 0.32. The free jet exhausts through a 56 in square nozzle into a $34 \mathrm{ft} \mathrm{long} \mathrm{test} \mathrm{cell} \mathrm{with} \mathrm{a} 17 \mathrm{ft} \times 17 \mathrm{ft}$ cross section. The floor, ceiling, and walls of the test cell are treated with fiberglass wedges. The acoustically treated flow collector is designed to improve flow recirculation effects in the test cell.
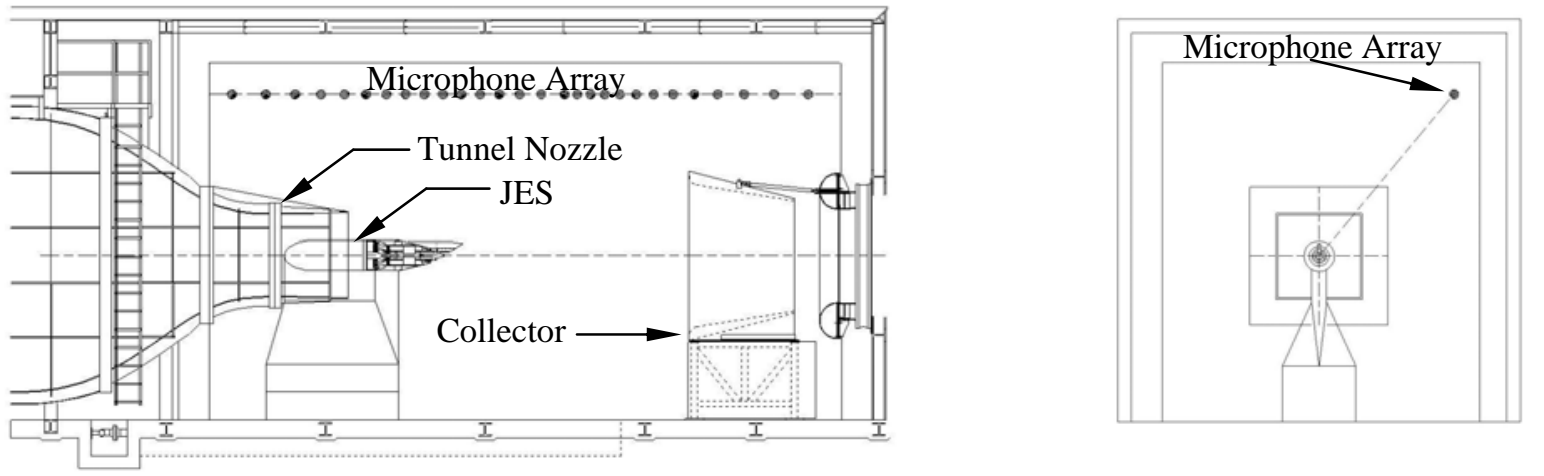

\section{Figure 1. A schematic of the Low Speed Aeroacoustics Wind Tunnel (LSAWT) and the Jet Engine} Simulator (JES).

The Jet Engine Simulator (JES) centered in the free stream jet consists of a coannular air stream used to accurately simulate engine nozzles systems. Each stream is equipped with a propane-fired, sudden-expansion burner and an electric pre-heater used to achieve real engine temperatures of commercial turbo-fan engines.

Acoustic measurements are made with a 28-element sideline microphone array (see Fig.1) located at $12 \mathrm{ft}$ from the centerline axis of the JES. The $1 \frac{1}{4}$ in diameter Bruel Kjaer type 4939 are used with the grid caps removed and calibrated with a piston phone and electrostatic calibrator. One-third octave data includes corrections for microphone calibration, wind tunnel background noise, and atmospheric absorption (corrected to the same reference day using the Shields and Bass ${ }^{6}$ method). The Amiet ${ }^{7}$ point source correction is used to account for acoustic propagation through the free jet shear layer. A Doppler shift is used for spectral data. The data is scaled to full scale and reported at a sideline distance of $1783 \mathrm{ft}$.

A representative $1 / 9^{\text {th }}$ scale, bypass ratio (BPR) 5 model system was used in the experiments. The baseline model consists of an externally plugged, 5.07 in diameter core nozzle with an exit area of $10.98 \mathrm{in}^{2}$ and a 9.45 in diameter fan nozzle with an exit area of $29.14 \mathrm{in}^{2}$. The baseline core nozzle had a uniform thickness of approximately 0.035 " at the trailing edge. (This baseline is different than that used in the previous fluidic chevron work of Henderson et al. ${ }^{4}$ and Kinzie et al. ${ }^{5}$, where the baseline core nozzle consisted of the fluidic chevron nozzle with no injection flow.) For the fluidic chevron configurations, the baseline core nozzle was replaced with one of 
four, second-generation fluidic chevron nozzles. These second-generation configurations introduced air through the pylon into contoured injection passages in the core nozzle (see Fig. 2). The injected air entered the core stream through six slots located near the nozzle trailing edge, spaced around the nozzle circumference. Four fluidic chevron nozzles were designed for the purpose of determining the effects of injection angle (angle between the injected flow and the core nozzle flow), slot length, and injection pressure ratio on the radiated noise. Two injection angles were used, called the steep angle and the shallow angle, with the former indicating an injection velocity more perpendicular to the direction of the core stream velocity. Three injector exit geometries were used, called the long slot, the short slot, and the perforated slot. The combinations of injection angle and slot geometry chosen for the four fluidic chevron core nozzles tested were: Configuration 1 - steep angle, short slot; Configuration 2 - shallow angle, short slot; Configuration 3 - steep angle, long slot; and Configuration 4 - shallow angle, perforated slot.

The total pressure of the injected air was controlled by a regulator and was measured along with its temperature in order to calculate the injected mass, momentum and velocity. For proprietary reasons, all the injected air properties reported are normalized by the maximum value obtained throughout the testing, and the details of the slot

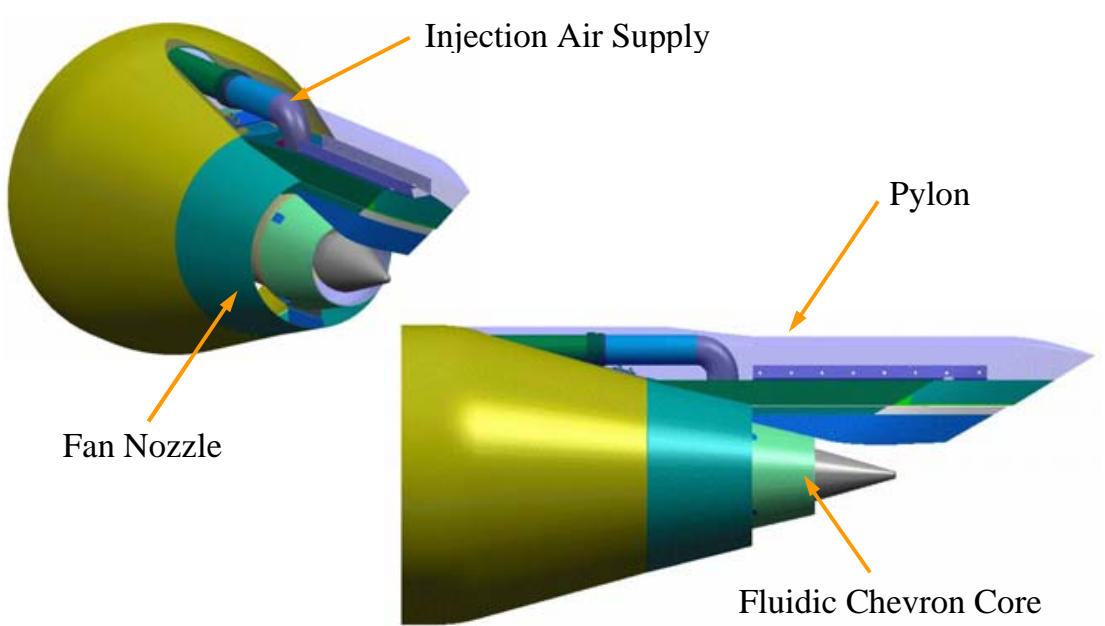

Figure 2. A schematic of the bypass ratio 5 model system used in the experiments. geometries are not given. The pylon was clocked at an angle of $135^{\circ}$ to the microphone array axis for all tests.

Data were acquired at representative takeoff and cutback power conditions although only data for takeoff power conditions with flight Mach numbers equal to 0.1 and 0.28 are presented here. In the experiments, the core nozzle pressure ratio (NPR, the ratio of the stagnation pressure to the ambient pressure), was equal to 1.56 and the total temperature was $1491{ }^{\circ} \mathrm{R}$. The fan NPR was equal to 1.75 and the total temperature was $647^{\circ} \mathrm{R}$. For each nozzle and operating condition, three or four replications of the data were taken.

\section{Results}

The first results presented give the effect of changing the injection pressure on the noise produced by the fluidic chevron nozzle Configuration 1, the steep angle short slot injector. Figure 3 gives the sideline directivities of the

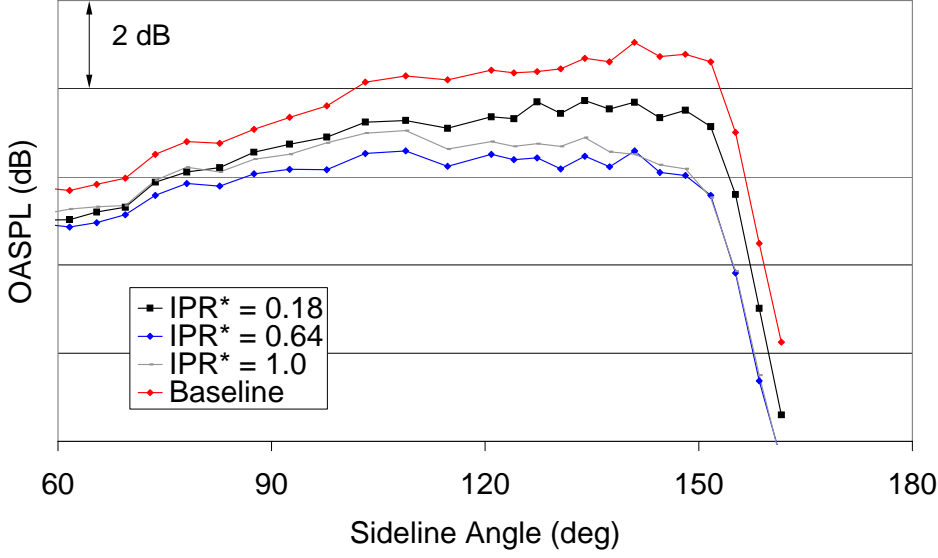

Figure 3. Sideline OASPL directivities for the fluidic chevron nozzle with steep injectors at a flight Mach number of 0.28 . baseline nozzle and Configuration 1 nozzle with three values of injection pressure (where IPR* is the ratio of the stagnation gage pressure to its maximum value). For the fluidic chevrons, the overall sound pressure levels at all angles decrease as the injection pressure increases, reaching a minimum OASPL within the range of IPR* tested. The 1/3 octave band acoustic spectra in Fig. 4 show that fluidic injection results in low frequency noise reduction from the baseline at observation angles of both $90^{\circ}$ and $140^{\circ}$ (the downstream peak jet noise direction). Also, higher injection pressures result in increased high frequency noise over that of the baseline nozzle at angles near $90^{\circ}$. However, it will be shown that the increased high frequency noise resulting from fluidic 


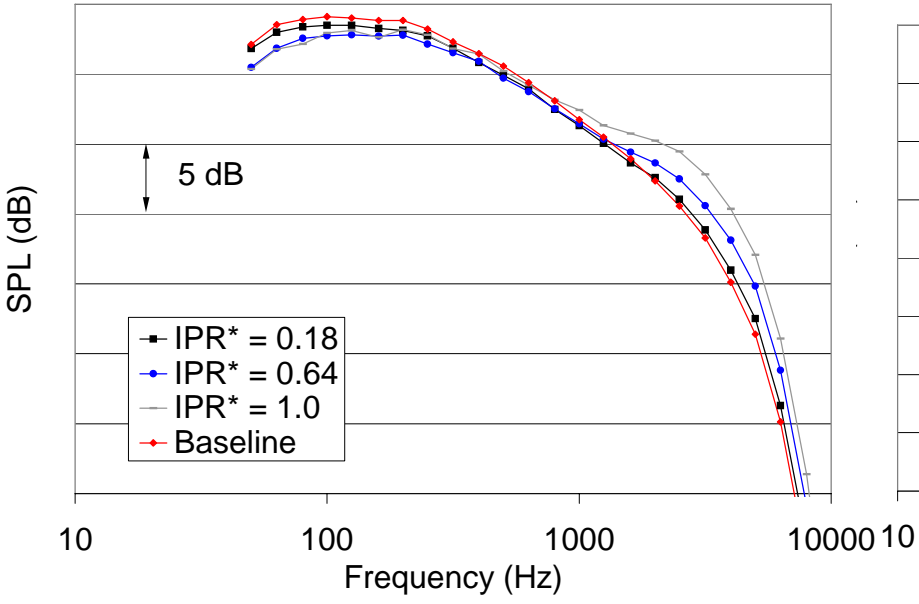

(a)

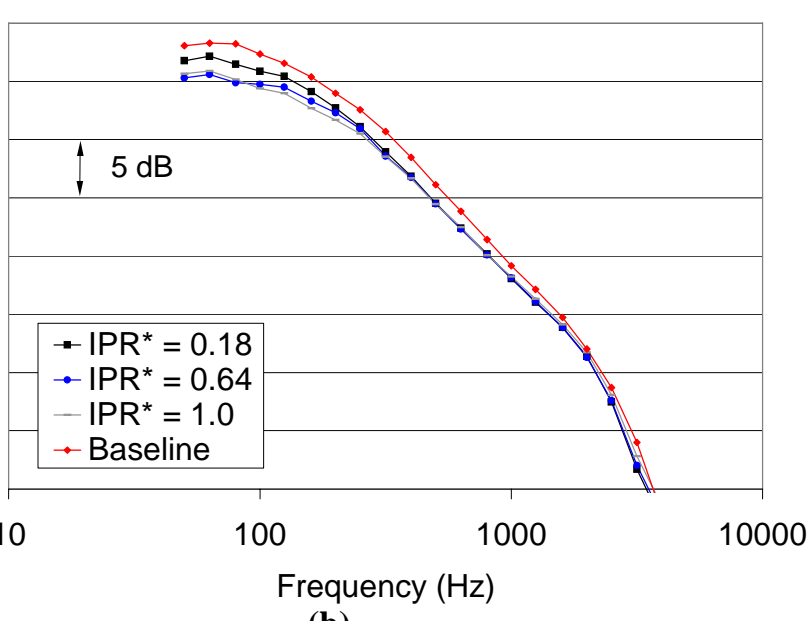

(b)

Figure 4. The $1 / 3$ octave band acoustic spectra for the fluidic chevron nozzle with steep injectors and the baseline nozzle at a flight Mach number of 0.28 and observation angles equal to (a) $90^{\circ}$ and (b) $140^{\circ}$.

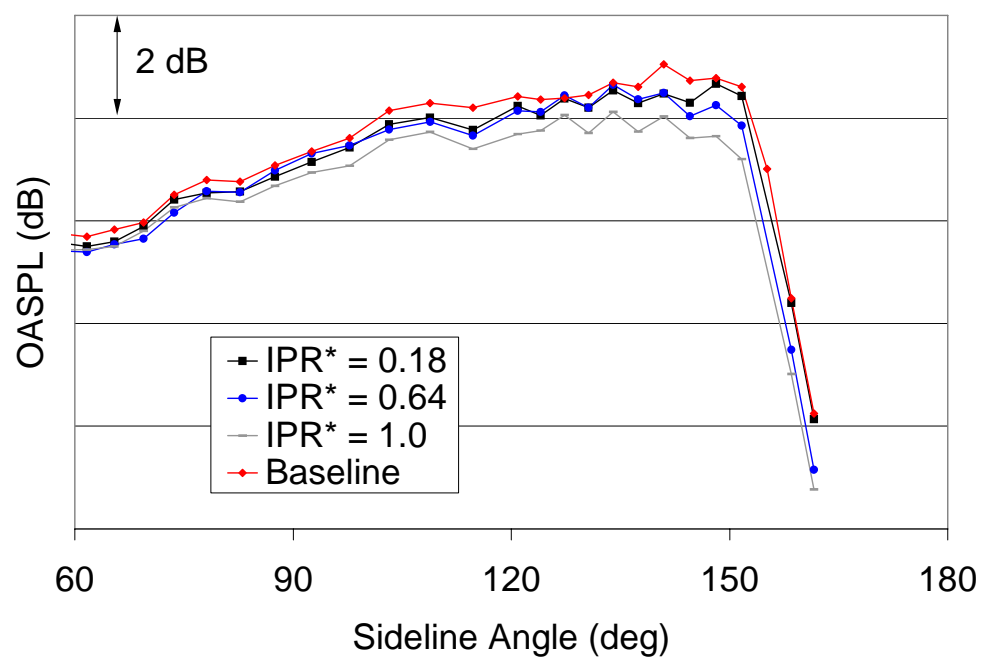

Figure 5. Sideline OASPL directivities for the fluidic chevron nozzle with shallow injectors compared to the baseline nozzle at a flight Mach number of 0.28 .

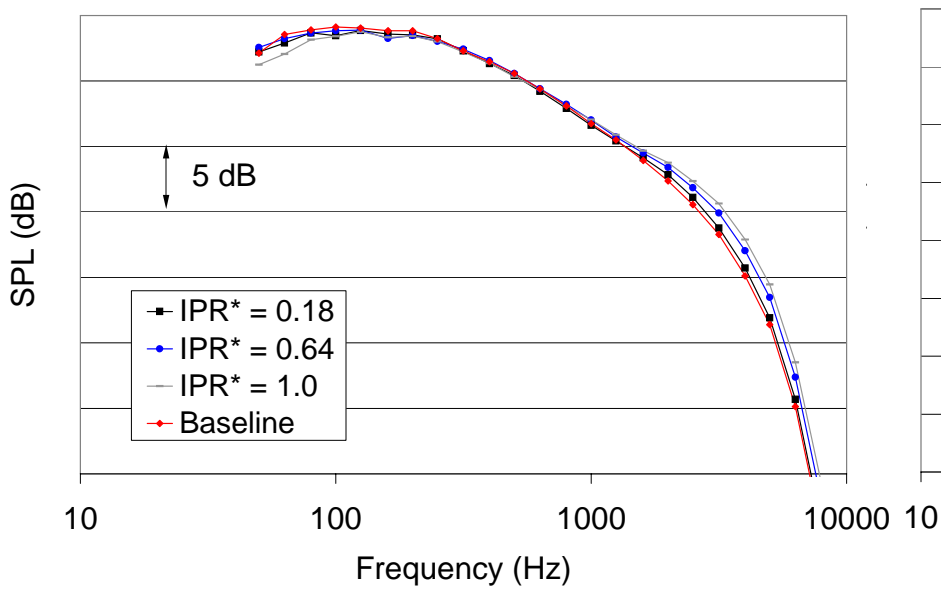

(a) injection with the second-generation nozzles is significantly reduced from that occurring with the first-generation nozzles.

The effect of steep verses shallow injection angles can be seen by comparing the results from the steep angle short slot injector nozzle to those of the shallow angle short slot injector nozzle (Configuration 2). The sideline directivity and spectra for the shallow injector are given in Figs 5 and 6 at the same IPR* as for the steep injector in Figs 3 and 4 . Although the trends are the same for the steep and shallow injectors, all noise changes at a given injector pressure are significantly smaller for the shallow injector than they are for the steep injector.

Figure 6. The 1/3 octave band acoustic spectra for the fluidic chevron nozzle with shallow injectors and the baseline nozzle at a flight Mach number of 0.28 and observation angles equal to (a) $90^{\circ}$ and (b) $140^{\circ}$. 
The effect of injection slot length on the radiated noise is seen by comparing results of Configurations 1 (steep angle short slot) and 3 (steep angle long slot). These results, given in figs 7 and 8, were obtained for equal injector mass flow, which required the injection pressure for the fluidic chevron with the long slot to be reduced from that

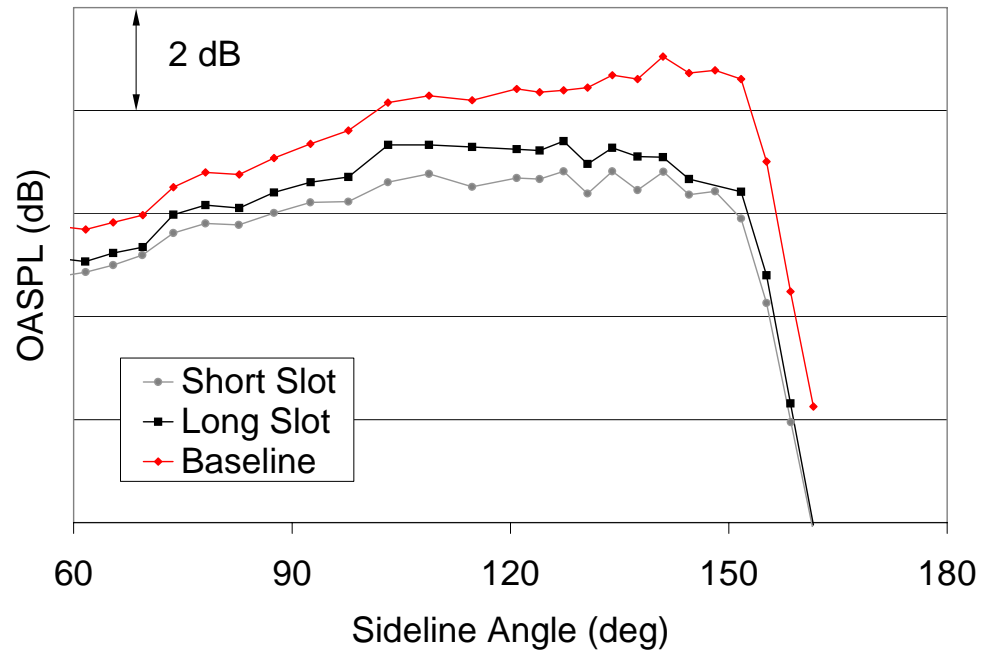

Figure 7. Sideline OASPL directivities for fluidic chevron nozzles with short and long injectors at the same mass flow rate, compared to the baseline nozzle. The flight Mach number is 0.28 .

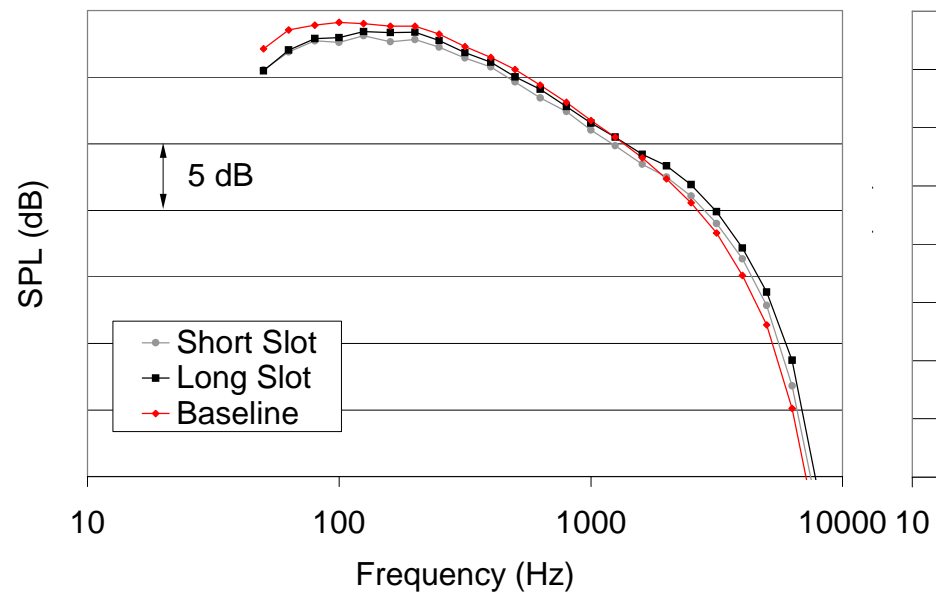

(a) for the fluidic chevron with the short slot. Figure 7 shows that increasing the slot length increases the overall sound pressure levels at all observation angles. For angles near the broadside of the jet [see Fig. 8 (a)], both fluidic chevrons produce very similar spectra although fluidic chevrons with short slots produce slightly lower sound pressure levels than fluidic chevrons with long slots. In the peak jet noise direction [see Fig. 8 (b)], the acoustic spectra for both fluidic chevrons are quite similar.

Comparisons between the directivities and the acoustic spectra of the current baseline nozzle, a firstgeneration fluidic chevron nozzle, and a second-generation fluidic chevron nozzle are shown in Figs. 9 and 10, respectively. The same injection pressures were used for both chevron nozzles. The improvements made to the second-

Figure 8. The 1/3 octave band acoustic spectra for chevron nozzles with short and long slot lengths and the baseline nozzle at a flight Mach number of 0.28 and observation angles equal to (a) $90^{\circ}$ and (b) $140^{\circ}$.

generation nozzles included thinner nozzle trailing edges, injection passages instead of slots cut into a common plenum, and injection ports that were moved in the downstream direction toward the nozzle trailing edges. The overall sound pressure levels (see Fig. 9) are similar for the first-generation and second-generation nozzles and show reductions in acoustic radiation over that of the baseline nozzle for all observation angles. For observation angles near the broadside of the jet (see Fig. 10), the improvements to the second-generation nozzle are shown to significantly reduce the high frequency acoustic radiation produced by the first-generation nozzle. The high frequency noise characteristics of the first-generation nozzles resulted in poor acoustic performance when evaluated on an effective perceived noise level basis. 


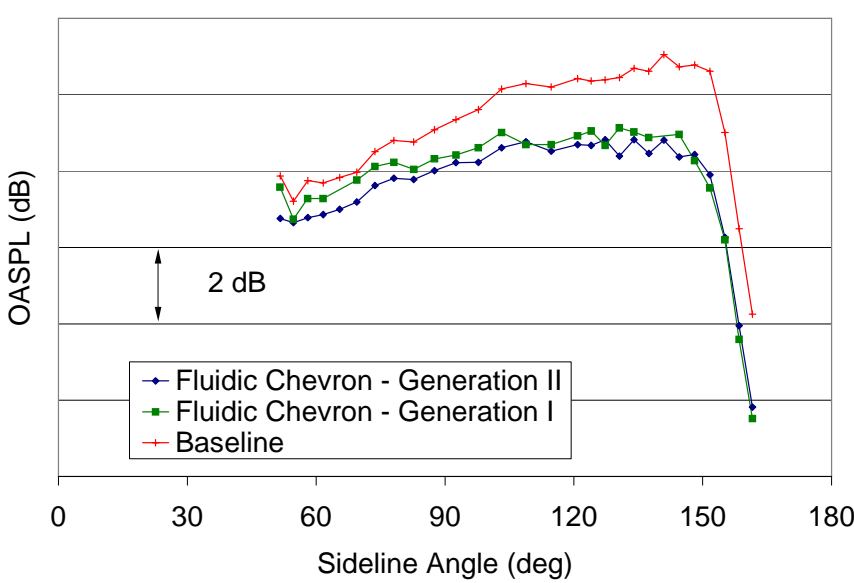

Figure 9. Sideline OASPL directivities for the best first-generation fluidic chevron, the best secondgeneration fluidic chevron, and the baseline nozzle at a flight Mach number of 0.28 . The IPR* is the same for both fluidic chevrons.

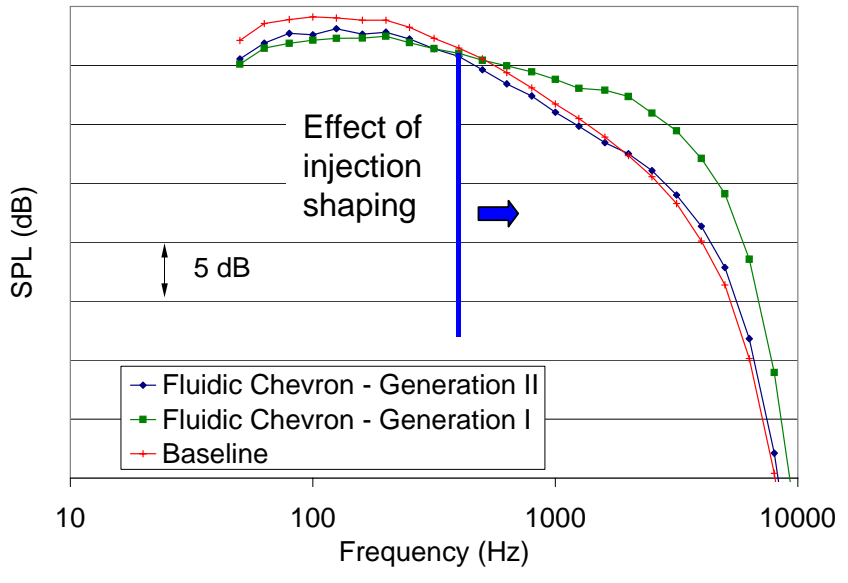

Figure 10. The acoustic radiation at an observation angle of $90^{\circ}$ for the nozzle configurations in Figure 9.

To reduce the effective perceived noise levels associated with the fluidic chevron nozzles, the maximum noise reduction at low frequencies in the peak jet noise direction must be achieved while controlling high frequency increases at broadside angles. The $80 \mathrm{~Hz}$ band at an observation angle of $134^{\circ}$ was chosen to evaluate low frequency noise characteristics and the $3160 \mathrm{~Hz}$ band at an angle of $90^{\circ}$ was chosen to evaluate the high frequency noise characteristics of the fluidic injectors. A plot of the sound pressure levels in the $80 \mathrm{~Hz}$ band at an observation

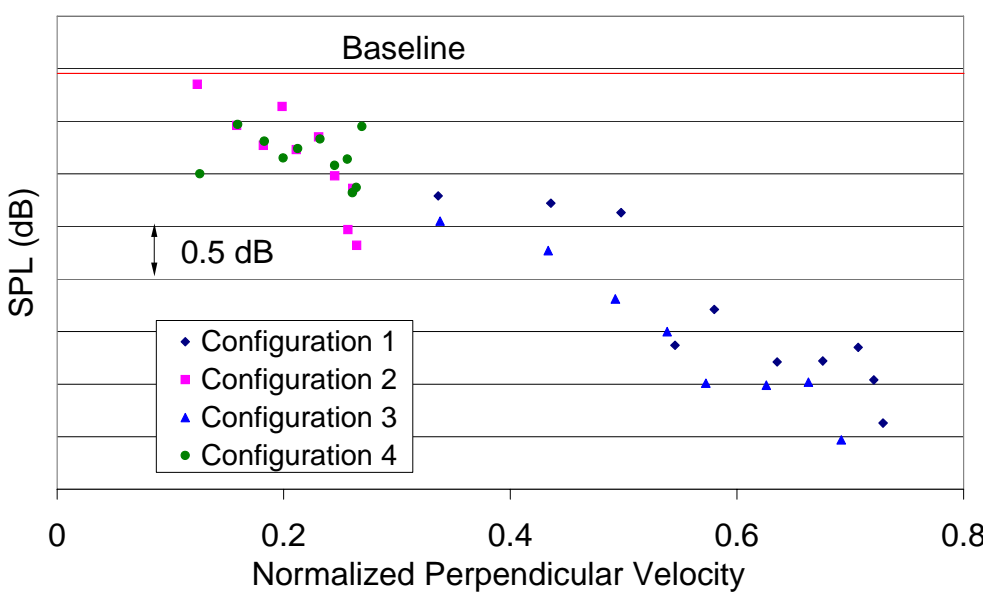

Figure 11. The sound pressure levels produced by all of the second-generation fluidic chevrons in the $80 \mathrm{~Hz}$ band at an observation angle of $134^{\circ}$ as a function of the component of the normalized injection velocity in a direction perpendicular to the core nozzle axis. The flight Mach number is $\mathbf{0 . 2 8}$. angle $134^{\circ}$ as a function of the component of the injection velocity in the direction perpendicular to the core nozzle flow is shown in Fig. 11, where the injection velocity has again been normalized by the maximum value achieved in the experiments. The four configurations shown in the legend represent the four different fluidic chevron nozzles used in the experiments. All fluidic chevron nozzles and all injection conditions resulted in lower sound pressure levels than that of the baseline nozzle. As shown in the plot, the low frequency sound pressure levels decrease as the injected velocity component increases. Although Greska and Krothapalli ${ }^{8}$ showed a reduction in overall sound pressure level with increasing injector momentum, the low frequency data from the present experiment did not scale well with either the momentum or the injection mass flow rate.

A plot of the sound pressure levels in the $3160 \mathrm{~Hz}$ band at an observation angle of $90^{\circ}$ is shown in Fig. 12 as a function of the injection flow momentum in a direction perpendicular to the core nozzle flow. The momentum has been normalized by the maximum momentum used in the experiments. (The data did not collapse as well when plotted against the injection mass flow rate or the injection velocity component perpendicular to the core nozzle axis.) While all injection nozzles and all injection conditions result in increased sound pressure levels over that of the baseline nozzle, reducing the perpendicular momentum reduces high frequency noise radiated in this direction.

Papamoschou and Hubbard ${ }^{9}$ showed that the penetration depth of a transverse supersonic jet into supersonic flow was related to the ratio of the momentum of the transverse jet to the momentum of the supersonic flow. The 


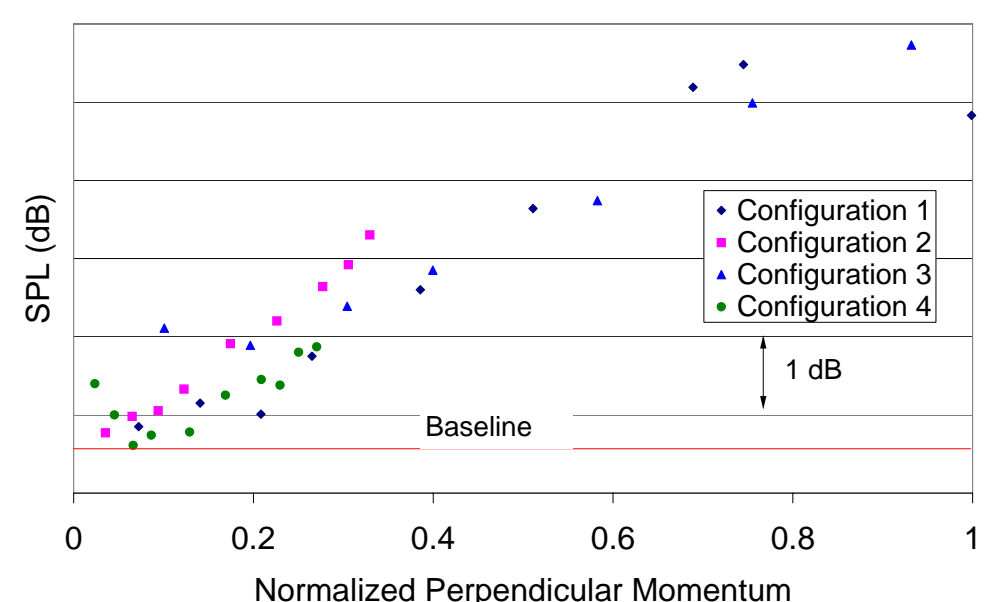

Figure 12. The sound pressure levels produced by all of the second-generation fluidic chevrons in the $3160 \mathrm{~Hz}$ band at an observation angle of $90^{\circ}$ as a function of the component of the normalized injection momentum perpendicular to the core nozzle flow direction. The flight Mach number is $\mathbf{0 . 2 8}$

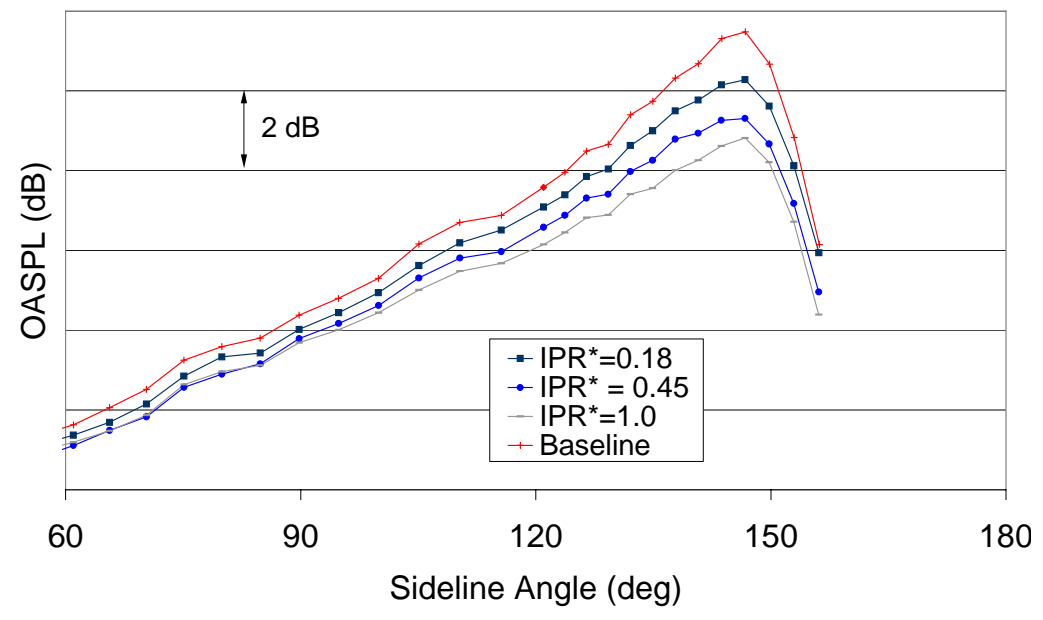

Figure 13. Sideline OASPL directivities for the fluidic chevron nozzle with steep injectors and the baseline nozzle at a flight Mach number of 0.10 . increases in high frequency noise with increasing injector momentum observed in Fig. 12 may be related to the injector penetration depth although increases in penetration depth appear to decrease low frequency noise ${ }^{8}$.

The effectiveness of the fluidic chevrons at lower flight Mach number can be seen in Figs 13 through 16 . These figures again present the sideline directivities and acoustic spectra for Configurations 1 and 2 at different IPR*, now at flight Mach number 0.10

The acoustic characteristics of the fluidic chevron nozzle with steep injectors at flight Mach number 0.10 (Figs 13 and 14) are seen to be similar to those at 0.28 (Figs 3 and 4). At both Mach numbers there is high frequency noise increase at $90^{\circ}$ and low frequency decrease at $140^{\circ}$, although these effects may be smaller than at Mach 0.28. The only difference in the trends between the two Mach numbers is that the increase in OASPL at highest injection pressure at Mach 0.28 is not present at Mach 0.10, due to the high frequency noise increases being more severe at the higher Mach number. Results for shallow injection at Mach 0.10 (Figs 15 and 16)are also similar to those at Mach 0.28 (Figs 5 and 6). The OASPL decreases with injection pressure are somewhat higher at Mach 0.10 , but this results only because the shape of the directivity of the jet noise changes with flight Mach number.

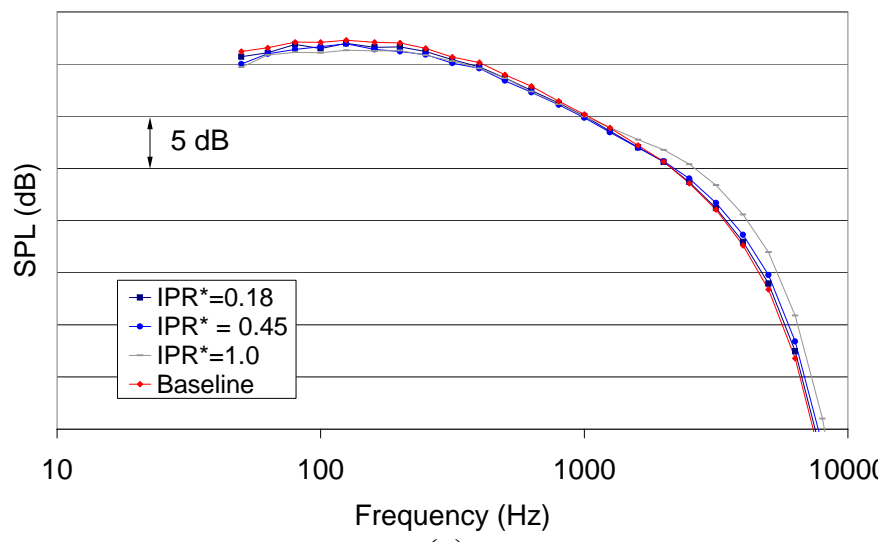

(a)

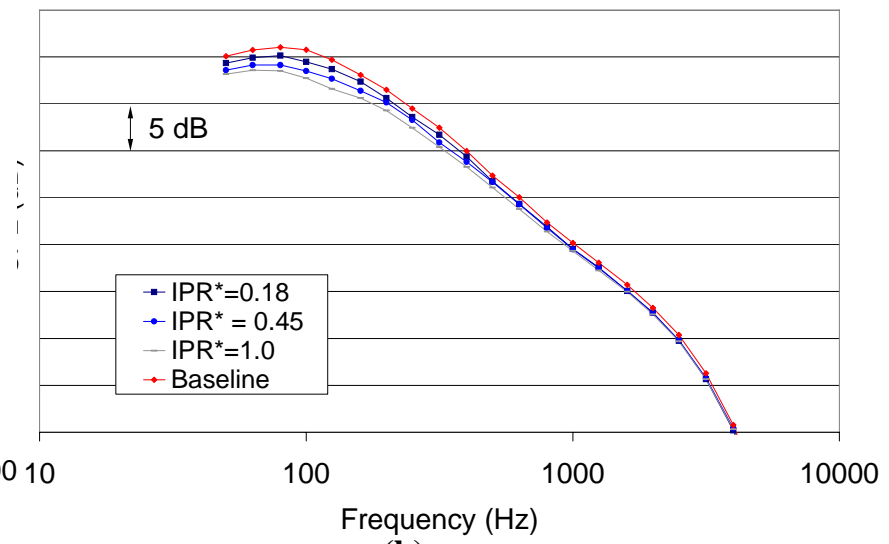

(b)

Figure 14. The 1/3 octave band acoustic spectra for fluidic chevrons with the steep injectors at a flight Mach number of 0.10 and observation angles equal to (a) $90^{\circ}$ and (b) $140^{\circ}$. 


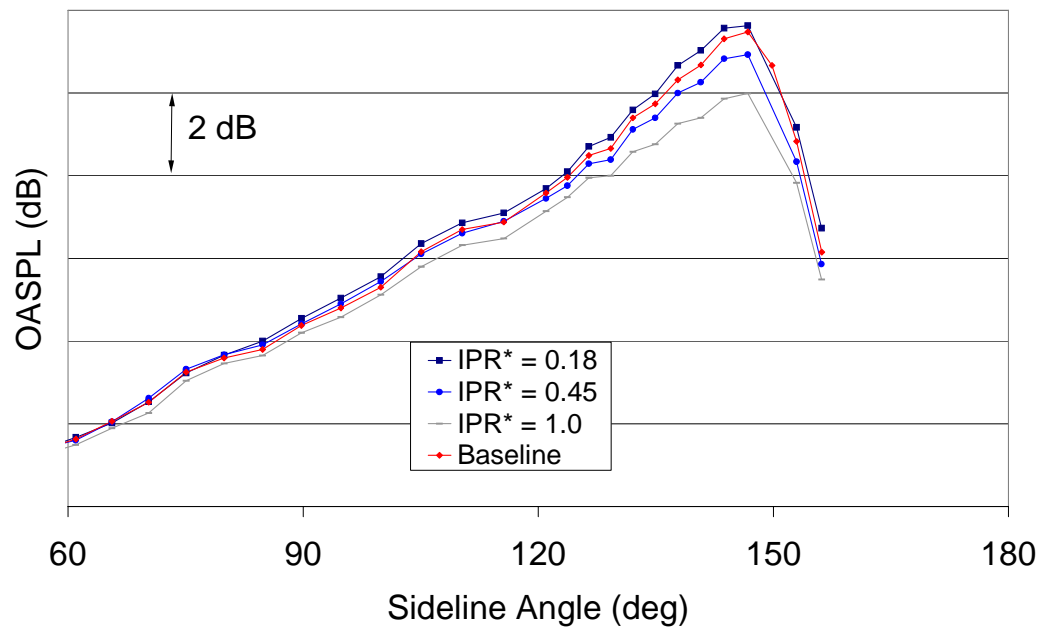

Figure 15. Sideline OASPL directivities for the fluidic chevron nozzles with shallow injectors and the baseline nozzle at a flight Mach number of 0.10

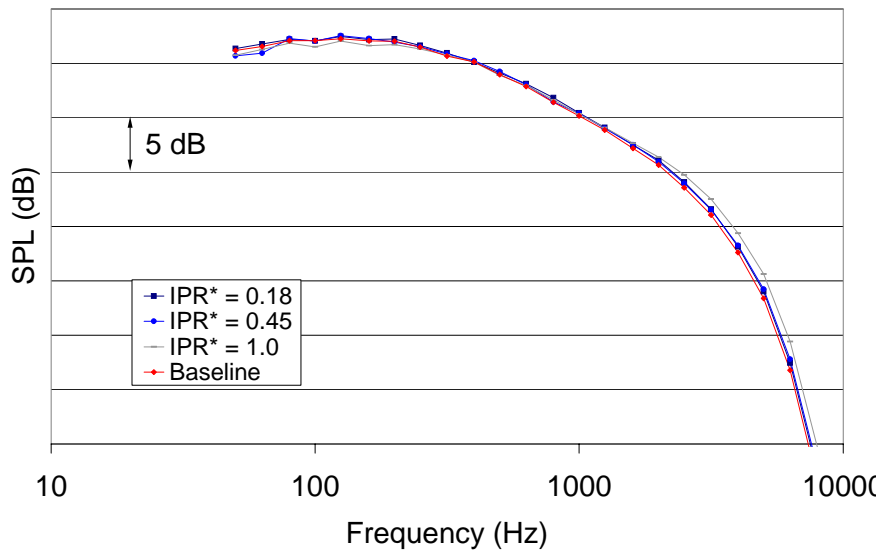

(a)
The sideline directivities for the best (in terms of lowest EPNL) secondgeneration fluidic chevron nozzle, the baseline nozzle, and a core mechanical chevron nozzle are shown in Fig. 17. The overall sound pressure levels produced by the mechanical chevron are slightly lower than those of the fluidic chevron at all observation angles although both chevron nozzles significantly reduce acoustic radiation over that of the baseline nozzle. The acoustic spectra at an observation angle of $90^{\circ}$ for the three nozzles are shown in Fig. 18. The spectra for the mechanical chevron and the fluidic chevron are quite similar although the mechanical chevron reduces low frequency noise more than the fluidic

Figure 16. The $1 / 3$ octave band acoustic spectra for the fluidic chevrons with shallow injectors at a flight Mach number of 0.10 and observation angles equal to (a) $90^{\circ}$ and (b) $140^{\circ}$.

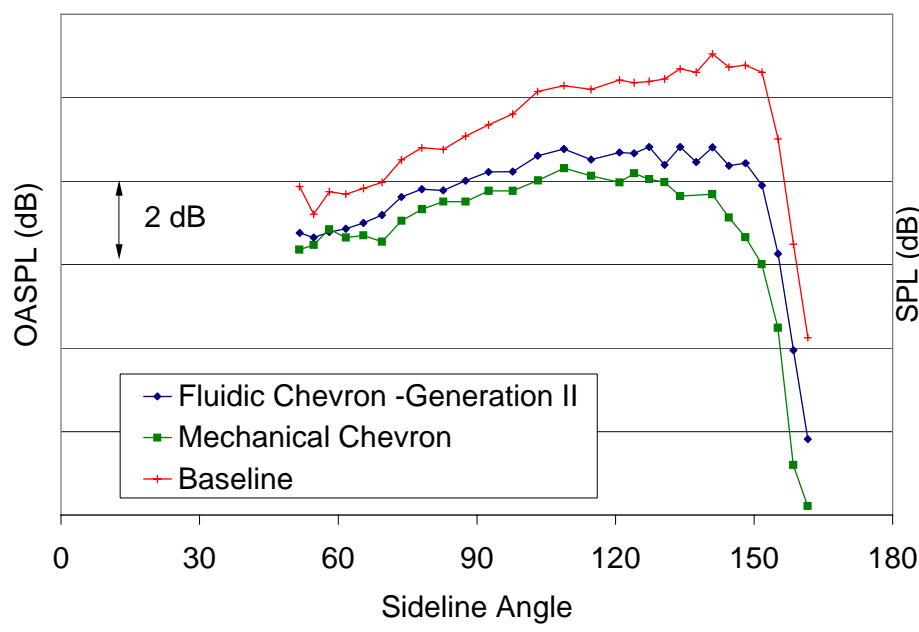

Figure 17. Sideline OASPL directivities for the best second-generation fluidic chevron nozzle, a core mechanical chevron nozzle, and the baseline nozzle at a flight Mach number of 0.10 .

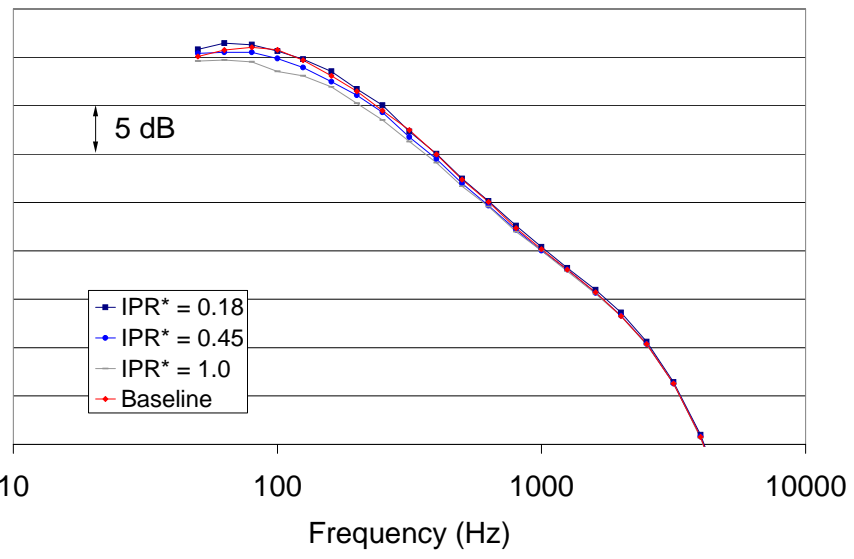

(b)

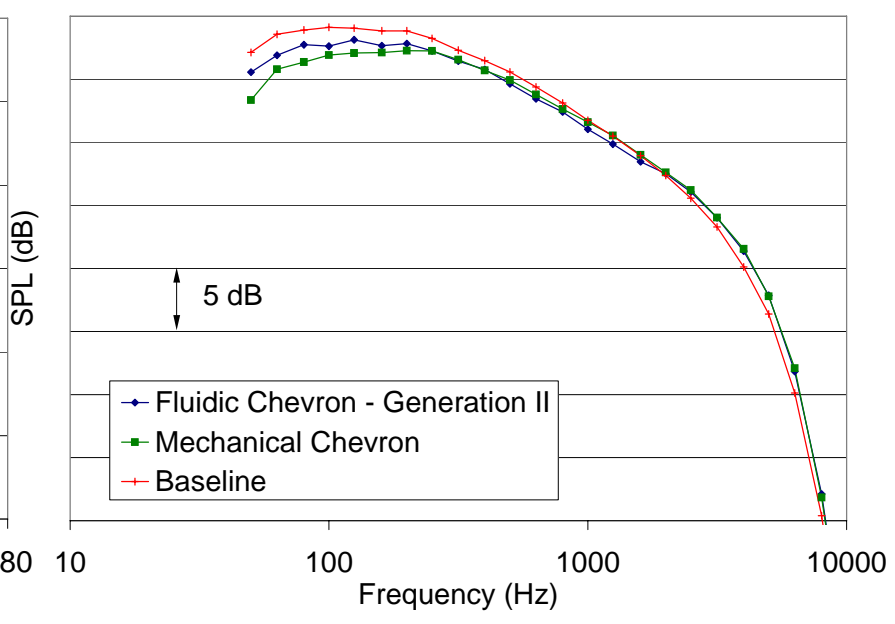

Figure 18. The acoustic radiation at an observation angle of $90^{\circ}$ for the mechanical chevron, fluidic chevron, and the baseline nozzle at a flight Mach number of 0.10 .

8

American Institute of Aeronautics and Astronautics 
Table 1. $\triangle$ EPNL

\begin{tabular}{|l|c|}
\hline Configuration & $\Delta$ EPNL \\
\hline Fluidic Chevron & 0.8 \\
\hline Mechanical Chevron & 1.1 \\
\hline
\end{tabular}

chevron. Computation of the reductions in effective perceived noise levels for the mechanical and fluidic chevron over that of the baseline nozzle are shown in Table 1. The effective perceived noise levels of the fluidic chevron nozzles are approaching those of the core mechanical chevrons after two generations of nozzles. Additional development of the fluidic injection technique may result in EPNL levels that equal or exceed those of the mechanical chevrons.

\section{Conclusion}

Improvements to the injection passages and nozzle trailing edges of the second-generation fluidic chevron nozzles have resulted in better acoustic performance than those of the first-generation nozzles. High frequency noise produced by the first-generation nozzles has been reduced with the second-generation nozzles and significant improvements in effective perceived noise levels have also been achieved. A comparison between the new fluidic chevrons and the core mechanical chevrons has shown that the acoustic characteristics of the fluidic chevrons are approaching those of the mechanical chevrons after two generations of nozzles.

Increased low frequency noise reduction in the peak jet noise direction is achieved with increased injection velocity in the direction perpendicular to the core nozzle flow and, therefore, injectors with steep injection angles demonstrate better low frequency noise characteristics than those with shallow injection angles for the same mass injection rate. High frequency noise increases at angles near the broadside of the jet scales with the momentum of the injected flow. To achieve acceptable effective perceived noise levels, the high frequency increases associated with increased momentum of the injected flow must be balanced with low frequency reductions associated with increased injected velocity. Future flow-field measurements with particle image velocimetry will be used to understand better the connection between the injection flow parameters and the physics of the jet plume.

\section{Acknowledgments}

The authors wish to thank the Jet Noise Laboratory staff for their many contributions to the planning and operation required for the experiment that produced the results written in this paper. Alan Douglas and Harry Haskin are also acknowledged for leading the mechanical design of the model system.

\section{References}

${ }^{1}$ Janarden, B.A., Hoff, G.E., Barter, J.W., Martens, S., Gliebe, P.R., Mengel, V., and Dalton, W.N., “AST critical propulsion and noise reduction technologies for future commercial subsonic engines," NASA/CR-2000-2100039, 2000.

${ }^{2}$ Saiyed, N., and Bridges, J., "Tabs and mixers for reducing low bypass ratio jet noise," AIAA-99-1986, Bellevue, WA, 1999.

${ }^{3}$ Martens, S., “Jet noise reduction technology development at GE Aircraft Engines,” ICAS 2002 Congress, pp. 842.1-842.10.

${ }^{4}$ Henderson, B.S., Kinzie, K.W., Whitmire, J., and Abeysinghe, A., "The impact of fluidic chevrons on jet noise, AIAA 20052888, Montery, CA, 2005

${ }^{5}$ Kinzie, K.W., Henderson, B.S., Whitmire, J., and Abeysinghe, A., "Fluidic chevrons for jet noise reduction," Proceedings of The 2004 International Symposium on Active Control of Sound and Vibration (ACTIVE 2004), INCE/USA, Williamsburg, VA, 2004.

${ }^{6}$ Shields, F. D. and Bass, H. E. “A study of atmospheric absorption of high frequency noise and application to fractional octave bands of noise,” NASA Contractor Report 2760, 1976.

${ }^{7}$ Amiet, R. K., "Correction of open jet wind tunnel measurements for shear layer refraction,” AIAA-77-54, 1977.

${ }^{8}$ Greska, B., and Krothapalli, A., "Jet noise reduction using aqueous microjet injection,” AIAA-2004-2971, 2004.

${ }^{9}$ Papamoschou, D., and Hubbard, D. G., "Visual observations of supersonic transverse jets,” Experiments in Fluids, Vol. 14, 1993, pp $468-476$. 